\title{
The distribution of carnitine and acetylcarnitine in the rabbit epididymis and the carnitine content of rabbit spermatozoa during maturation
}

\author{
E. R. Casillas and Surang Chaipayungpan \\ Department of Chemistry, New Mexico State University, Las Cruces, New Mexico 88003, \\ U.S.A.
}

\begin{abstract}
Summary. The highest levels of carnitine and acetylcarnitine were found in the cauda, and spermatozoa from the proximal cauda contained significantly greater amounts of carnitine than those removed from the corpus or caput epididymidis. Acetylcarnitine levels (as a \% of the total carnitine pool) were greater in all regions of the rabbit epididymis than has been reported in other species. It is suggested that the accumulation of carnitine is involved in sperm maturation.
\end{abstract}

\section{Introduction}

Carnitine is present in exceedingly high concentrations in the epididymis of rats (Marquis \& Fritz, 1965a; Casillas, 1972; Brooks, Hamilton \& Mallek, 1974) and other species (Casillas, 1972). We have previously shown that carnitine accumulates in bovine spermatozoa as they mature in the epididymis and have suggested that one of the functions of the organ is to supply carnitine to spermatozoa as they mature (Casillas, 1973). We have also presented evidence that carnitine plays a special role in the metabolism of mature bovine and monkey spermatozoa (Casillas \& Erickson, 1975a). The relation between the carnitine content of epididymal spermatozoa and their ability to fertilize eggs has not been studied previously and is one of the subjects of this report. Since the fertility of spermatozoa removed from various sections of the rabbit epididymis is known (Bedford, 1966; Orgebin-Crist, 1967), we decided to measure the carnitine content of rabbit epididymal spermatozoa and to compare these data with the fertility of spermatozoa removed from the same regions of the epididymis.

In addition, we measured carnitine levels present in sections of the epididymis to determine whether the distribution pattern of carnitine within the rabbit epididymis is similar to that of other species. Finally, since acetylcarnitine has been proposed as an energy source for rabbit spermatozoa as they traverse the epididymis (Storey \& Keyhani, 1974), we have measured the levels of this compound in rabbit epididymal tissues. The idea proposed by Lardy \& Phillips (1941a, b) that endogenous phospholipids serve as an energy source for spermatozoa as they traverse the epididymis is generally accepted but has been recently questioned (Darin-Bennett, Poulos \& White, 1973; Storey \& Keyhani, 1974). Storey \& Keyhani (1974) have reported that the mitochondria of rabbit epididymal spermatozoa lack type I carnitine palmitoyl transferase and hence are unable to oxidize long-chain fatty acids. Since these mitochondria readily oxidize acetylcarnitine (Storey \& Keyhani, 1974), acetylcarnitine in epididymal fluids might serve as an important energy source for spermatozoa.

\section{Materials and Methods}

\section{Preparation of epididymal and sperm extracts}

Epididymides from mature New Zealand White rabbits, 1-3 years old and weighing 3-4.5 kg, were obtained from Pel-Freez Biological, Inc., Rogers, Arkansas, U.S.A. After killing the animals, the organs were removed within $4 \mathrm{~min}$ and were placed on ice for shipment to our 
laboratory where they arrived within $24 \mathrm{~h}$ and were processed immediately. In preliminary experiments, epididymides were removed from freshly killed New Zealand White rabbits and were processed as described below. Comparisons between shipped organs and fresh organs showed no differences in sperm motility, viability or carnitine content. Furthermore, no differences were observed in the carnitine or acetylcarnitine content of various regions of the epididymis.

The epididymides were carefully trimmed of adipose and connective tissue and of superficial blood vessels and were divided into 6 sections (Text-fig. 1). The sections were pooled and then lacerated with a sharp scalpel and were incubated at $37^{\circ} \mathrm{C}$ and gently stirred in a medium containing $20 \mathrm{~mm}-\mathrm{NaH}_{2} \mathrm{PO}_{4}, 119 \mathrm{mM}-\mathrm{NaCl}, 5 \mathrm{~mm}-\mathrm{KCl}, 2 \mathrm{mM}-\mathrm{MgCl}_{2}$ (pH 7.0) for $30 \mathrm{~min}$. The spermatozoa which were released into the medium were collected by centrifuging the suspension at $1000 \mathrm{~g}$ for $5 \mathrm{~min}$. The spermatozoa were then washed twice in 10 volumes of the buffered salt medium before being analysed for carnitine content. Microscopic examination of sperm preparations from all regions showed them to be at least $95 \%$ pure. Sperm counts were performed by conventional haemocytometer techniques and purity was estimated by comparing the number of spermatozoa obtained during cell-counting procedures to the total number of cells observed in the same field. The original supernatant solution from the incubation was combined with the supernatant solutions obtained from the washes and this fraction (referred to as epididymal fluid) was analysed for carnitine and acetylcarnitine content.

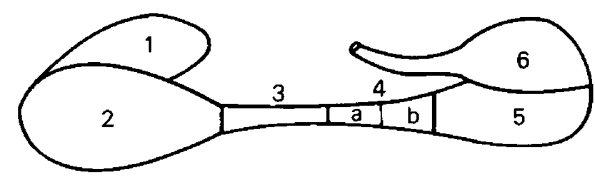

Text-fig. 1. Diagrammatic representation of the rabbit epididymis showing the regions used in the study: 1, proximal caput; 2 , distal caput; 3 , proximal corpus; 4 , distal corpus (subdivided into $4 \mathrm{a}$ and $4 \mathrm{~b}) ; 5$, proximal cauda; 6 , distal cauda epididymidis.

\section{Analysis of carnitine and acetylcarnitine}

Extracts for the analyses of carnitine and acetylcarnitine were prepared according to the procedure described by Pearson \& Tubbs (1964) as modified by Casillas (1972). In brief, spermatozoa suspended in $0.5 \mathrm{M}^{-} \mathrm{HClO}_{4}$ were disrupted further by sonic irradiation. The epididymal fluid solutions were acidified by the addition of sufficient $5 \mathrm{M}-\mathrm{HClO}_{4}$ to yield a final concentration of $0.5 \mathrm{M}$. In both cases, acid-insoluble material was removed by centrifugation and the acid-soluble fraction was neutralized with $\mathrm{KOH}$. The $\mathrm{KClO}_{4}$ precipitate was removed by another centrifugation and carnitine present in the clear, neutral extracts was determined by the method described by Cederblad \& Lindstedt (1972) with modifications suggested by McGarry \& Foster (1976). The limit of sensitivity was $5 \mathrm{pmol}$ and the relative deviation of 5 duplicate analyses was $2-10 \%$. In 3 experiments, the recovery of carnitine added to perchloric acid extracts of spermatozoa was $100 \pm 10 \%$. Acetylcarnitine, present in the $\mathrm{HClO}_{4}$-soluble fraction, was estimated by assaying for carnitine liberated by the hydrolytic procedure described by Pearson \& Tubbs (1964). Comparative studies that measured acetylcarnitine by a direct method (Marquis \& Fritz, 1965a) showed that essentially all of the carnitine liberated by hydrolysis was originally acetylcarnitine.

\section{Materials}

Carnitine acetyltransferase from pigeon breast muscles (98 units/mg protein) was obtained from Sigma Chemical Co., St. Louis, Missouri; L-carnitine chloride was from Supelco Inc., Bellefonte, Pennsylvania; [acetyl-1-14 C]acetyl Coenzyme A (sp. act. $52 \mathrm{mCi} / \mathrm{mmol}$ ) and [acetyl${ }^{3} \mathrm{H}$ ]acetyl Coenzyme A (sp. act. $1.97 \mathrm{Ci} / \mathrm{mmole}$ ) were from New England Nuclear, Boston, 
Massachusetts; Dowex $2 \times 8$ (200-400 mesh) was from Biorad Laboratories, Richmond, California; sodium tetrathionate was from ICN Pharmaceuticals, Plainview, New York. All other reagents were of the best grade available from commercial sources.

\section{Results}

\section{Carnitine content of epididymal spermatozoa}

The carnitine content of spermatozoa removed from the 6 regions studied is shown in Table 1. There was a significant increase in sperm carnitine content as the cells passed from the midpiece to the cauda. Small amounts of acetylcarnitine, less than $10 \%$ that of carnitine, were also present in epididymal spermatozoa.

Table 1. Carnitine content ( $\mathrm{nmol} / 10^{8}$ spermatozoa) of rabbit spermatozoa removed from various regions of the epididymis (see Text-fig. 1)

\begin{tabular}{lcccccc}
\hline & \multicolumn{7}{c}{ Region } \\
\cline { 2 - 7 } & 1 & 2 & 3 & 4 & 5 & 6 \\
\hline $\begin{array}{l}\text { Content } \\
\text { No. of spermatozoa } \\
\times 10^{8} \text { analysed }\end{array}$ & $5 \cdot 2 \pm 1 \cdot 1$ & $6.5 \pm 1 \cdot 8$ & $4.9 \pm 0.9$ & $5 \cdot 4 \pm 1 \cdot 6$ & $12 \cdot 4 \pm 0 \cdot 5^{*}$ & $13 \cdot 1 \pm 0 \cdot 2^{*}$ \\
\hline
\end{tabular}

Values are mean \pm s.e.m. for 10 separate experiments on about 34 epididymides per experiment.

* Significantly different from values in Regions $1,2,3$ and $4(P<0 \cdot 05$, Student's $t$ test).

Since significant numbers of fertile spermatozoa have been found in the distal corpus epididymidis (Bedford, 1966; Orgebin-Crist, 1967) we investigated whether cells removed from the proximal and distal halves from this section (Region 4) contained different levels of carnitine. The trend of values obtained from 4 separate experiments, with 34 epididymides per experiment, suggest that spermatozoa from Region $4 \mathrm{~b}$ contain more carnitine $\left(7.8 \pm 2.2 \mathrm{nmol} / 10^{8}\right.$ spermatozoa) than do spermatozoa removed from Region $4 \mathrm{a}\left(4.4 \pm 0.2 \mathrm{noml} / 10^{8}\right.$ spermatozoa), although the difference was not statistically significant.

\section{Distribution of carnitine and acetylcarnitine}

The distributions in various segments of the epididymis are shown in Table 2. The data represent the amount of non-sperm carnitine and acetylcarnitine that was washed out of the segments by lacerating them and incubating them in a salt-buffer. Results from preliminary

Table 2. Distribution of carnitine and acetylcarnitine in rabbit epididymal tissue

\begin{tabular}{|c|c|c|c|c|c|}
\hline \multirow[b]{2}{*}{ Region } & \multirow[b]{2}{*}{$\begin{array}{c}\text { Mean wt } \\
\text { (g) }\end{array}$} & \multicolumn{2}{|c|}{ Carnitine } & \multicolumn{2}{|c|}{ Acetylcarnitine } \\
\hline & & $\begin{array}{c}\mathrm{nmol} / \\
\text { epididymis }\end{array}$ & $\begin{array}{c}\mathrm{nmol} / \\
\mathrm{g} \text { wet wt }\end{array}$ & $\begin{array}{c}\text { nmol/ } \\
\text { epididymis }\end{array}$ & $\begin{array}{c}\text { nmol/ } \\
\text { g wet wt }\end{array}$ \\
\hline 1 & 0.118 & $31 \cdot 9 \pm 3 \cdot 1$ & $349 \pm 46$ & $16 \cdot 1 \pm 3 \cdot 4$ & $96 \pm 16$ \\
\hline 2 & 0.125 & $52.5 \pm 4.5$ & $540 \pm 74$ & $36 \cdot 3 \pm 9 \cdot 4$ & $197 \pm 24$ \\
\hline 3 & 0.053 & $36.0 \pm 7.6$ & $760 \pm 56$ & $15 \cdot 3 \pm 4 \cdot 9$ & $157 \pm 55$ \\
\hline 4 & 0.079 & $44 \pm 9.2$ & $667 \pm 91$ & $17 \cdot 2 \pm 6.9$ & $138 \pm 34$ \\
\hline 5 & 0.35 & $372 \pm 39$ & $2320 \pm 404$ & $180 \pm 38$ & $536 \pm 75$ \\
\hline 6 & 0.23 & $215 \pm 17$ & $1669 \pm 196$ & $155 \pm 32$ & $481 \pm 36$ \\
\hline
\end{tabular}

Values are mean \pm s.e.m. from 34 epididymides/experiment and 15 and 8 separate experiments for the analysis of carnitine and acetylcarnitine respectively. 
experiments showed that this procedure extracted at least $90 \%$ of the total carnitine and acetylcarnitine present in the segments when total carnitine was measured by extracting the segments with dilute perchloric acid (Pearson \& Tubbs, 1964). Furthermore, less than $5 \%$ of the total carnitine from the cauda and less than $1 \%$ from the caput epididymidis was present in washed spermatozoa. The fact that the compounds were easily washed from incised tissue suggests that they were derived from the epididy mal plasma rather than the tissue itself.

\section{Discussion}

The results presented here extend our previous observation (Casillas, 1973) that carnitine accumulates in bovine spermatozoa as they progress through the epididymis. These results are qualitatively similar to those found in the bull (Casillas, 1973) in which carnitine levels increased about 10 -fold as the cells passed from the caput to the cauda epididymidis. The carnitine content of spermatozoa removed from various regions of the rabbit epididymis correlates well with the fertility of these cells as reported by Bedford (1966) and by Orgebin-Crist (1967). These investigators have reported that although significant numbers of fertile spermatozoa first appear in the distal corpus, the cauda region contains a higher percentage of mature spermatozoa than does the distal corpus. For example, Orgebin-Crist (1967) found that after intrauterine insemination, spermatozoa from the distal corpus epididymidis fertilized $57 \%$ of the recovered eggs while caudal spermatozoa fertilized $90 \%$. We have not shown that the carnitine content of spermatozoa from the distal corpus, whose fertility is appreciable, is significantly greater than that of spermatozoa from the proximal corpus epididymidis. Nevertheless, there appears to be an increase in the carnitine content of spermatozoa as they progress through this region. Another suggestion of a close correlation between the accumulation of carnitine by rabbit spermatozoa and their fertility comes from the results of preliminary experiments now in progress on spermatozoa maintained in culture within sections of the epididymis. We have observed that the carnitine content of spermatozoa from the proximal corpus, maintained in culture with dihydrotestosterone, is greater than that of control cells in culture without androgen (E. R. Casillas, unpublished). Orgebin-Crist \& Tichenor (1973) have reported that under identical conditions of culture, spermatozoa develop the ability to fertilize ova. Obviously, further experimentation is necessary to determine whether the accumulation of carnitine is an indispensable event in the maturation of spermatozoa.

The increase in sperm carnitine content may also be related to the development of sperm motility that takes place during transit through the epididymis. In the rabbit, spermatozoa gradually change their pattern of motility from a weak vibration of the tail in cells released from the seminiferous tubules to a vigorous unidirectional progression in cells released from the cauda epididymidis (Bedford, 1975). We have previously postulated that the high carnitine content of mature spermatozoa together with an active carnitine acetyltransferase serves to buffer against changes in the acetylation state of Coenzyme A (Casillas \& Erickson, 1975a), and this metabolic role for carnitine may enable mature spermatozoa to synthesize ATP at a rate sufficient to support vigorous motility.

The results presented here compare favorably with those of Brooks et al. (1974) who reported that $87.2,9.6$ and $3.2 \%$ of the total carnitine in the rat cauda epididymidis was recovered in the epididymal plasma, in washed spermatozoa, and in the remaining tissue, respectively. The observation that most of the rabbit epididymal carnitine is found in the cauda epididymidis (Regions 5 and 6) agrees with similar results obtained in the rat (Marquis \& Fritz, 1965a; Casillas, 1972; Brooks et al., 1974) and in the monkey, boar and bull (Casillas, 1972).

The total amounts of carnitine plus acetylcarnitine in the rabbit, about $1.2,1.7$ and 5.0 $\mu \mathrm{mol} / \mathrm{g}$ wet weight in the caput, corpus and cauda epididymidis, respectively, are about the same as those found in the monkey (Casillas, 1972), about twice those found in the bull (Casillas, 1972) and about one-third to one-half the amounts present in the rat (Brooks et al., 1974). 
Surprisingly, acetylcarnitine in the rabbit epididymis makes up a larger percentage of the $\mathrm{HClO}_{4}$ soluble carnitine fraction than is found in other species: in the rabbit the value ranges from about $30 \%$ in the corpus to $40 \%$ in the caput and cauda epididymidis compared with $25 \%$ in the caput to $6 \%$ in the cauda of the bull (Casillas, 1972) and about $10 \%$ in both the caput and cauda of rats (Brooks et al., 1974). The high levels of acetylcarnitine in the rabbit epididymis suggest that it might be used as an energy source, at least in this species, by spermatozoa as they progress through the epididymis. Whether these differences between species are due to differences in the rates of acetylcarnitine synthesis or in the rates of acetylcarnitine oxidation by spermatozoa is unknown and is a problem that is being investigated. That intact epididymal spermatozoa are capable of oxidizing the acetyl group of acetylcarnitine has been demonstrated, at least with bovine cells. When caudal spermatozoa were incubated with $\left[{ }^{14} \mathrm{C}\right.$-acetyl]acetylcarnitine in vitro, ${ }^{14} \mathrm{CO}_{2}$ was produced at a rate of $30 \mathrm{nmol} / \mathrm{h} / 10^{9}$ cells.

We have previously shown that rat epididymal spermatozoa and epididymal tissue lack the ability to synthesize carnitine (Casillas \& Erickson, 1975b). Tanphaichitr \& Broquist (1974) have shown that the final reaction in the synthesis of carnitine occurs primarily in the liver and to a lesser extent in the testis. The testis does not appear to be the source of epididymal carnitine since near-normal levels of the compound accumulate in the epididymides of castrated rats that have been treated with testosterone (Marquis \& Fritz, 1965b; Casillas \& Erickson, 1975b). It is therefore suggested that the carnitine reaches the epididymis via the blood supply and that the concentration of carnitine within epididymal fluids and the accumulation of carnitine by spermatozoa are an integral part of epididymal function.

This work was supported by grants from the National Institutes of Health (HD 10664, RR 08136).

\section{References}

Bedford, J.M. (1966) Development of the fertilizing ability of spermatozoa in the epididymis of the rabbit. J. exp. Zool. 163, 319-330.

Bedford, J.M. (1975) Maturation, transport, and fate of spermatozoa in the epididymis. In Handbook of Physiology, vol. 5, pp. 303-317, Eds D. W. Hamilton \& R. O. Greep, American Physiological Society, Washington, D.C.

Brooks, D.E., Hamilton, D.W. \& Mallek, A.H. (1974) Carnitine and glycerylphosphorylcholine in the reproductive tract of the male rat. $J$. Reprod. Fert. 36, 141-160.

Casillas, E.R. (1972) The distribution of carnitine in male reproductive tissues and its effect on palmitate oxidation by spermatozoal particles. Biochim. Biophys. Acta 280, 545-551.

Casillas, E.R. (1973) Accumulation of carnitine by bovine spermatozoa during maturation in the epididymis. J. biol. Chem. 248, 8227-8232.

Casillas, E.R. \& Erickson, B.J. (1975a) The role of carnitine in spermatozoan metabolism: substrateinduced elevations in the acetylation state of carnitine and coenzyme-A in bovine and monkey spermatozoa. Biol. Reprod. 12, 275-283.

Casillas, E.R. \& Erickson, B.J. (1975b) Studies on carnitine synthesis in the rat epididymis. J. Reprod. Fert. 44, 287-291.

Cederblad, B. \& Lindstedt, S. (1972) A method for the determination of carnitine in the picomole range. Clin. Chim. Acta 37, 235-243.

Darin-Bennett, A., Poulos, A. \& White, I.G. (1973) A re examination of the role of phospholipids as energy substrates during incubation of ram spermatozoa. $J$. Reprod. Fert. 34, 543-546.

Lardy, H.A. \& Phillips, P.H. (1941a) The interrelation of oxidative and glycolytic processes as sources of energy for bull spermatozoa. Am. J. Physiol. 113, $602-609$.

Lardy, H.A. \& Phillips, P.H. (1941b) Phospholipids as a source of energy for motility of bull spermatozoa. Am. J. Physiol. 135, 542-548.

Marquis, N.R. \& Fritz, I.B. (1965a) The distribution of carnitine, acetylcarnitine, and carnitine acetyltransferase in rat tissues. $J$. biol. Chem. 240, 21932196.

Marquis, N.R. \& Fritz, I.B. (1965b) Effects of testosterone on the distribution of carnitine, acetylcarnitine, and carnitine acetyltransferase in tissues of the reproductive system of the male rat. $J$. biol. Chem. 240, 2197-2200.

McGarry, J.D. \& Foster, D.W. (1976) An improved and simplified radioisotopic assay for the determination of free and esterified carnitine. J. Lipid Res. 17, 277280.

Orgebin-Crist, M.C. (1967) Maturation of spermatozoa in the rabbit epididymis: fertilizing ability and 
embryonic mortality in does inseminated with epididymal spermatozoa. Annls Biol. anim. Biochim. Biophys. 7, 373-389.

Orgebin-Crist, M.C. \& Tichenor, P.L. (1973) Effect of testosterone on sperm maturation in vitro. Nature, Lond. 245, 328-329.

Pearson, P.J. \& Tubbs, P.K. (1964) Tissue levels of acidinsoluble carnitine in rat heart. Biochim. Biophys. Acta 84, 772-773.
Storey, B.T. \& Keyhani, E. (1974) Energy metabolism of spermatozoa. II. Comparison of pyruvate and fatty acid oxidation by mitochondria of rabbit epididymal spermatozoa. Fert. Steril. 25, 857-864.

Tanphaichite, V. \& Broquist, H.P. (1974) Site of carnitine biosynthesis in the rat. $J$. Nutr. 104, 16691673.

Received 24 July 1978 